UDC 92:51

PACS 01.65. $+\mathrm{g}$

DOI: $10.22363 / 2658-4670-2021-29-4-306-336$

\title{
In Memory of Vladimir Gerdt
}

\author{
Victor F. Edneral ${ }^{1,2}$ \\ ${ }^{1}$ Skobeltsyn Institute of Nuclear Physics \\ Lomonosov Moscow State University \\ 1 (2), Leninskie Gory, Moscow, 119991, Russian Federation \\ 2 Peoples' Friendship University of Russia (RUDN University) \\ 6, Miklukho-Maklaya St., Moscow, 117198, Russian Federation
}

(received: August 13, 2021; accepted: September 22, 2021)

This article is a memorial, it is dedicated to the memory of the head of the Scientific Center for Computational Methods in Applied Mathematics of RUDN, Professor V.P. Gerdt, whose passing was a great loss to the scientific center and the computer algebra community. The article provides biographical information about V. P. Gerdt, talks about his contribution to the development of computer algebra in Russia and the world. At the end there are the author's personal memories of V. P. Gerdt.

Key words and phrases: computer algebra, quantum computing, mimetic methods, polynomial computer algebra methods

\section{Introduction}

The name of Vladimir Gerdt is widely known among computer algebra community. Many years he was a professor at the Joint Institute for $\mathrm{Nu}$ clear Research (JINR), where he was the head of the Group of Algebraic and Quantum Computations (http://compalg.jinr.ru/CAGroup), and an organizer of many mathematical conferences. A few years ago, he was invited to head the Scientific Center for Computational Methods in Applied Mathematics founded in RUDN university. His passing was a great loss to the entire community.

\section{Biography}

Vladimir Gerdt was born in Engels near Saratov. He earned his M. Sc. in Theoretical Physics from Saratov State University in 1971, his Ph. D. in Theoretical and Mathematical Physics from JINR in 1976, and his D. Sc. in Mathematics and Computer Science from JINR in 1992. In 1997 he got the scientific title Professor in Mathematics and Computer Science by 
specialty "Application of Computer Techniques, Mathematical Modelling and Mathematical Methods to Scientific Research".

After his M. Sc. Vladimir Gerdt worked in JINR until his death in January 5, 2021. He began as an engineer-programmer (1971-1975), then he worked as a junior researcher (1975-1977) at the JINR Department of Radiation Safety where a software for neutron spectroscopy was developed. In 1977 he moved to the JINR Laboratory of Computing Techniques and Automation renamed in 2000 as Laboratory of Information Technologies, where he worked as a researcher (1977-1980) and as a senior researcher (1980-1983), and since 1983 as the head of the research group on computer algebra. Vladimir Gerdt worked abroad for several years, in Lille and Aachen, using Russian, English, German and French in his work.

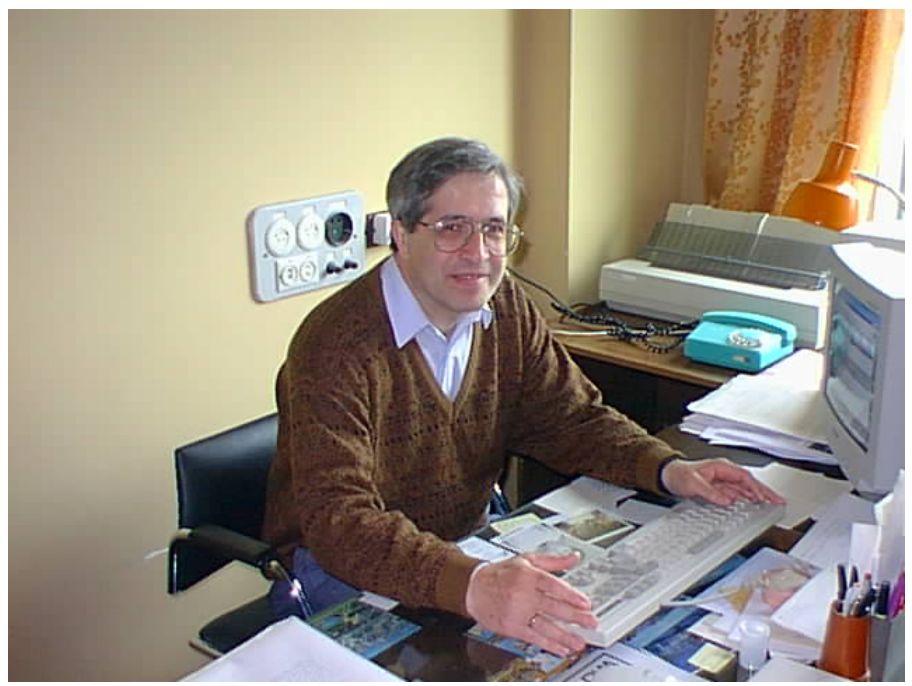

Figure 1. Vladimir Gerdt in his office. Dubna, 1998

\section{Professional activities}

V. Gerdt prepared 243 scientific articles, he edited 10 books. His latest researches are devoted to the construction of involutive monomial bases and to the discretizations of incompressible Navier-Stokes equations. His last huge article was published in ArXiv in September 2020.

Vladimir was the referee at journals and organizations:

- Journal of Symbolic Computation;

- Programming and Computer Software;

- Physics of Particles and Nuclei Letters;

- Russian Foundation for Basic Research;

- Russian Science Foundation.

Vladimir was a member of:

- Association for Computing Machinery (ACM);

- ACM Special Interest Group on Symbolic and Algebraic Manipulation (SIGSAM); 
- Editorial Board of Journal of Symbolic Computation (Academic Press);

- Advisory Board of Computer Science Journal of Moldova;

- Special Computer Algebra Group of German Societies on Computer Science.

Vladimir took part in the coordination of the international research projects:

- he was adjoint coordinator of the INTAS-93-0030 project "Computer Algebra, Symbolic and Combinatorial Tools in Differential Algebra and Differential Equations, with impact in Fundamental Physics and Control Theory" with 10 research teams from EC countries and 7 research teams from NIS countries;

- scientific coordinator of cluster A: Computer Assisted Mathematics of the INTAS-93-0893 project "ERSIM-FSU Cooperative Network in Informatics and Applied Mathematics" with 10 research teams in EC countries and 10 research teams from NIS countries.

Vladimir Gerdt paid great attention to teaching. He gave 24 lecture courses for students and young scientists. Under his supervision 10 master theses were prepared, $9 \mathrm{Ph}$. D. theses were defended. He was the scientific consultant of Yuri Blinkov's thesis for Doctorship of Sciences.

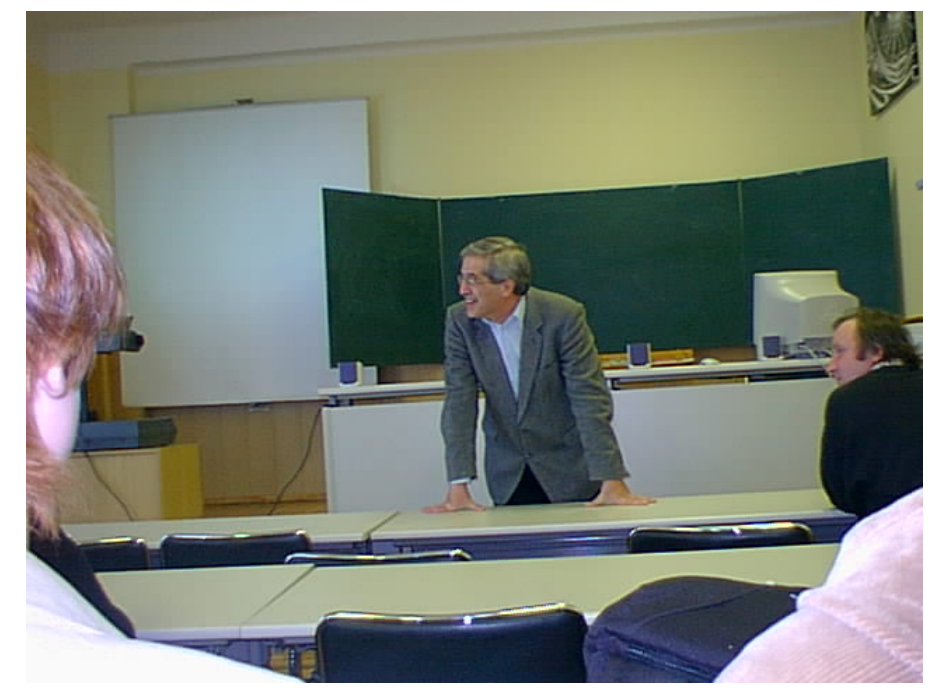

Figure 2. Vladimir with students. Dubna, 2002

\section{Vladimir Gerdt and computer algebra}

\subsection{At the beginning of computer algebra}

Vladimir was one of the first who started computer algebra usage in the USSR in the 70th. This activity was supported by Academic Dmitry Shirkov and Professor Nikolay Govorun.

In the early 80th the Joined Institute for Nuclear Research (JINR, Dubna) bought the computer CDC-6500. It was powerful enough for the implementation of the universal computer algebra systems. Professor Tony Hearn kindly 
passed the REDUCE system to the JINR during his visit to Dubna. Professor Gerdt with colleagues took a large part in its implementation in the institute and assisted in spreading the REDUCE in the scientific centers of the USSR.

Vladimir got the "First JINR Prize (1986) for the Research on Installation, Development and Application of Program Systems for Symbolic Computation on Mainframe Computers".

Vladimir was on Committees of many conferences. The main of them are:

- International Symposium on Symbolic and Algebraic Computation (ISSAC);

- Conference on Applications of Computer Algebra (ACA);

- Polynomial Computer Algebra (PCA);

- Computer Algebra in Scientific Computing (CASC), Vladimir was one of its founders. Now CASC-2021 is the 23rd conference in this series. It takes place in Sochi (Russia).

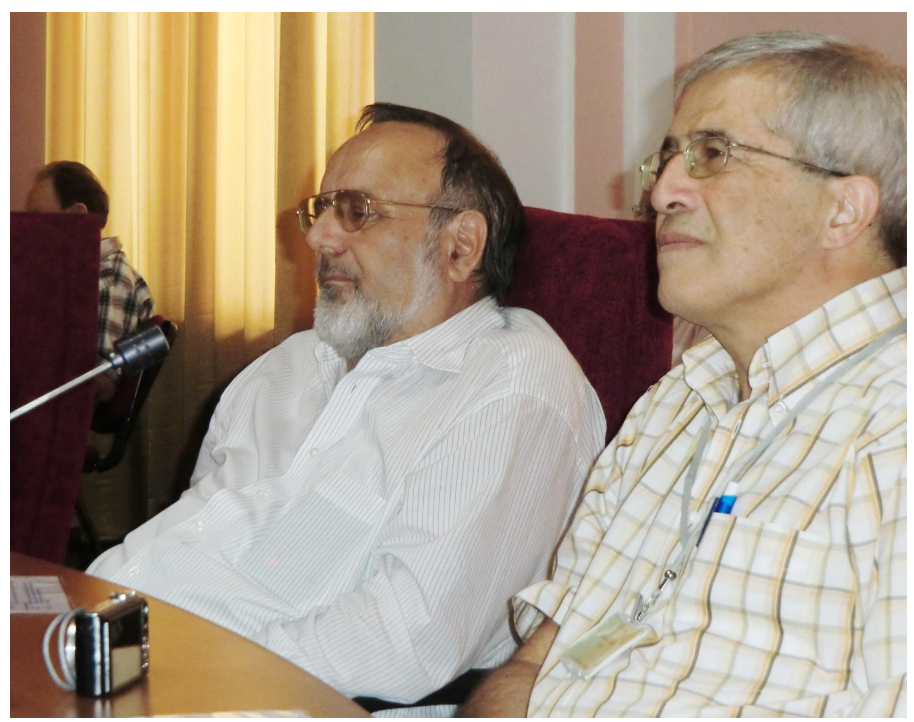

Figure 3. Foundators of the CASC Profs. Vladimir Gerdt and Ernst Mayr, Armenia, 2010

\subsection{Partial differential equations}

A large cycle of works by Vladimir Gerdt was devoted to the study of the compatibility of systems of partial differential equations (PDEs) by means of computer algebra. The key to solving the problem was the CauchyKovalevskaya theorem, which reduces the study of the solvability of some classes of systems of partial differential equations to the study of the compatibility of a system of algebraic equations for the coefficients of the corresponding power series.

Theoretical research on the compatibility of systems of nonlinear differential equations in general form was started at the beginning of the 20th century by Riquier [1], Janet [2], and Thomas [3]. V. P. Gerdt told us about the long months he spent in the 1980s searching and studying these far from well-known works written in various European languages. 
Riquier proposed a complete ordering for partial derivatives, using which he distinguished some of the derivatives, called principal ones, with respect to which the system of PDEs can be resolved. The remaining derivatives, called parametric, leave arbitrariness in the solution and affect the setting of the initial conditions. As a result, a theory was constructed containing the CauchyKovalevskaya theorem as a special case. Along the way of algorithmization of these results, Janet introduced the partition of independent variables into multiplicative and non-multiplicative for the principal derivatives. Thomas generalized the Riquier-Janet approach over the case of nonlinear algebraic equations with respect to the principal derivative. He showed how to check the consistency of a system or to split it into subsystems in a finite number of steps (Thomas decomposition).

These works, at first, gave rise to a modern theory, which makes it possible to investigate the compatibility of systems of partial differential equations and carry out their decomposition into subsystems (Thomas decomposition), created by V.P. Gerdt together with D. Roberts and very elegantly inscribed in the theory of differential rings. Later, the theory was used to create the DifferentialThomas package, recently implemented in Maple (https: //www.maplesoft.com). A monograph by D. Roberts is devoted to this issue [4].

\subsection{Polynomial computer algebra}

One of the most important achievements of algebra in the XX century was the creation of the theory of Gröbner bases, which made it possible to study problems from the theory of polynomial rings and algebraic geometry using a computer [5]. The main obstacle to the application of this technique is the cost of calculating these bases according to the Buchberger algorithm, therefore, the development of more efficient methods for finding Gröbner bases has been and remains an urgent problem of computer algebra. The key idea of the theory of Gröbner bases is the division of a polynomial into polynomials generating a certain ideal $J$. In the case of the ring $\mathbb{Q}[x]$ every ideal is principal and any polynomial $g$ can be uniquely divided by the polynomial $f$ generating the ideal $(f)$. In the case of the ring $\mathbb{Q}\left[x_{1}, \ldots, x_{n}\right]$ one can also talk about dividing the polynomial into polynomials $f_{1}, \ldots, f_{r}$ generating the ideal $J$, but this was realized only in the middle of the XXth century. One of the very first steps in introducing the operation of division of polynomials was to define the admissible McCauley ordering [6]. In the process of such division, expressions of the form $h f_{i}$ are successively subtracted from $g$, where $h \in \mathbb{Q}\left[x_{1}, \ldots, x_{n}\right]$, so that the leading coefficients in $g$ are canceled. As a result, instead of $g$, a new polynomial $g^{\prime}$ is obtained, the degree of which is less than the degree of $g$ and $g-g^{\prime} \in J$. Unfortunately, in the case of many variables, $g^{\prime}$ is uniquely determined in this way only when a special basis is chosen, the Gröbner basis, and only in this special basis it follows from $g \in J$ that $g^{\prime}=0$.

A wonderful idea proposed by V.P. Gerdt is that the division of a polynomial into polynomials generating the ideal $J$ can be made unambiguous if we preserve an additional structure on the set of monomials $M$, which he called involutive division. The concept of involutive division is closely related to the partitioning of independent variables introduced by Janet (see above) in the study of the compatibility of systems of partial differential equations. 
V.P. Gerdt brought ideas that arose in the theory of PDEs to polynomial algebra, which made it possible to look at old problems in a completely new light.

Involutive division allows for any monomial $m$ choosing one of the monomials of a given finite set $U$ and thus uniquely determine the choice of $h f_{i}$ when dividing $g$ by $\left(f_{1}, \ldots, f_{r}\right)$. The remainder of the division can now be called the normal form of the polynomial $g$ with respect to the polynomials $f_{1}, \ldots, f_{n}$. However, checking $g \in\left(f_{1}, \ldots, f_{r}\right)$ is reduced to checking $g^{\prime}=0$ not for every basis $f_{1}, \ldots, f_{r}$, but only for an involutive basis whose principal monomials satisfy certain properties, found and described by V.P. Gerdt and his disciple Yu. A. Blinkov. The first concrete example of involutive division was described by A. Yu. Zharkov, another disciple of V.P. Gerdt [7]-[10]. Soon V. P. Gerdt and Yu. A. Blinkov constructed many other involutive divisions. This made it possible to formulate a fundamentally new algorithm for constructing Gröbner bases [11]-[19].

Under the leadership of V.P. Gerdt, his disciples Yu. A. Blinkov and D. A. Yanovich created a number of algorithms and programs for calculating involutive bases of ideals of polynomial rings, including the open-source software package GINV (http://invo.jinr.ru). Their algorithms for calculating involutive bases appeared to be faster than Buchberger's algorithm and able to compete with the algorithms optimized by Fougeres and his disciples. The theory of involutive bases itself has become an important branch of computer algebra, to which the participants of international conferences on computer algebra regularly devote their articles and reports.

\subsection{Mimetic methods for solving partial differential equations}

Power series expansions are a very poor method for finding solutions to compatible systems of differential equations, except in the rare case when the solution is interesting in a small neighborhood of a given point. The main and, by and large, the only method for solving such systems is the finite difference method, according to which the system of differential equations in $\mathbb{R}^{n}$ is reduced to an infinite system of algebraic equations for the values of the sought functions at the grid nodes. Research in the field of finite-difference approximations of differential equations inheriting their basic properties has more than 60 years of history. Discretizations inheriting certain properties of continuous (differential) equations are called mimetic or compatible [20]-[25]. Discussing reports at conferences, we often heard from V.P. Gerdt about the importance of this concept for the development of numerical methods of mathematical modeling, as well as about the flexibility of the concept of inheritance, allowing for different interpretations.

In the last century, the transition from differential equations to algebraic ones was done by hand. V.P. Gerdt and Yu. A. Blinkov proposed a new approach in which this transformation was performed in computer algebra systems. The studies mentioned above have stimulated interest to the question of what happens to the differential consequences of discretization. V. P. Gerdt singled out a class of strongly consistent difference schemes. The property of strong consistency means not only the approximation of the original differential equations by the finite-difference scheme, but also the approximation of any algebraic consequence of these equations by the algebraic consequence of the 
difference equations that make up the scheme. These consequences include, in particular, local conservation laws.

V.P. Gerdt in his recent studies, carried out together with Yu. A. Blinkov and D. Roberts, strove to show the advantages of S-compatible schemes over others. For the demonstration, they have chosen one the system of NavierStokes equations, one of the most complex systems of great importance for applications. For this system, an S-compatible difference scheme was constructed and numerous computer experiments were carried out. A multipage report on this work by V.P. Gerdt was published in ArXiv [26] shortly before his death. One can only regret that the size of this study will not allow it to be published entirely as a full journal article.

\subsection{Applications of polynomial computer algebra methods in generalized Hamiltonian dynamics}

The use of involutive methods in applied, engineering and physical problems described by systems of underdetermined and overdetermined differential equations has become an area of special interest for V.P. Gerdt in the early 1990s. In mechanical systems, the configuration and phase spaces of which are subject to constraints and restrictions, evolution problems inevitably require involutive analysis. This is especially true for physical systems which possess a degenerate Lagrange function and are described in the framework of the generalized Dirac Hamiltonian dynamics. In a large cycle of works carried out by V.P. Gerdt together with colleagues from Bulgaria (D. Mladenov), Georgia (S. Gogilidze, A. Khvedelidze) and Moldova (Yu. Paliy), an algorithm was developed and applied for finding a complete set of constraints (of the first and second kind) for polynomial mechanical systems with a degenerate Lagrange function, which is based on the ideas of the theory of Gröbner bases and involutive division of polynomials. The efficiency of the proposed algorithm was demonstrated, in particular, when calculating the constraints in the so-called mechanical $S U(3)$ Yang-Mills model on a light cone, where, thanks to the use of computer calculations, for the first time it was possible to determine and classify the complete set of constraints inherent in the model.

\subsection{Applications of polynomial computer algebra methods in quantum theory}

Computational problems related to the description of quantum systems became another area of application of Gröbner bases in the studies of V.P. Gerdt. In the 21st century, quantum theory has ceased to be just a purely fundamental physical theory. It has acquired the status of the basic element of a new quantum technological design. These changes gave rise to new computationally intensive tasks. One of these problems is the problem of classifying quantum systems in terms of their quantum resource, in particular, depending on the complete set of characteristics responsible for the phenomenon of entanglement of quantum states. The joint research performed in Dubna in the period from 2006 to 2016 by V. P. Gerdt, Y. Paliya and A. Khvedelidze focused on this class of problems.

In these works, the algebraic structure of the ring of polynomial invariants of basic composite binary quantum systems, such as qubit-qubit and qubitqutrite pairs, was studied. As these studies have shown, computer algebra 
methods allow performing labor-intensive computational calculations and, thereby, determine the quantum resource of low-dimensional quantum systems, which is interesting from the point of view of various applications, including the theory of quantum information.

\section{Personal memories}

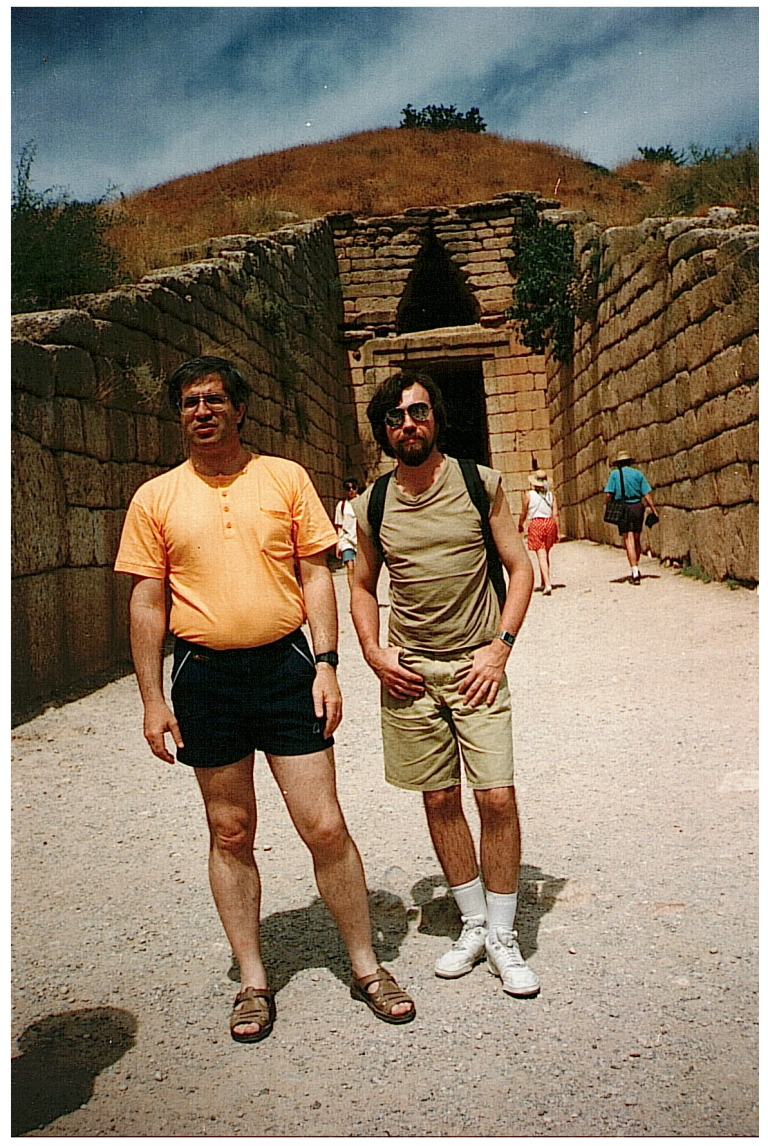

Figure 4. Vladimir Gerdt and Victor Edneral at Schliemann's excavations. Peloponnese, 1995

We met Vladimir Gerdt at the 3rd international conference on computer algebra and its applications in theoretical physics, which took place in September 1985 in Dubna. Later, we met at a couple of dozen conferences, at seminars, at defenses. I soon noticed that he took great care of those who were with him. Vladimir never spoke badly about anyone. He criticized, of course, but only in specific cases. A distinctive feature of Vladimir was great respect for people, for each person. And people felt it. It should be added, that Vladimir was a believer and observed orthodox church fasts and rituals. Without advertising it in any way. 
Vladimir had wide erudition and organized very interesting excursions for conference participants in amazing places. We were with him in Peloponnese, in Germany, in France, in Japan, in Spain, in Israel, in China e.t.c.

I do not remember who said "Where the captain is, there is the captain's bridge". This is about Vladimir. He was quickly becoming the soul of any company, he saw any task in every detail and imagined the roles of everybody.

A clear mind allowed him to ask wonderful questions during conference reports. Always to the point. Without any self-promotion. He was very humble.

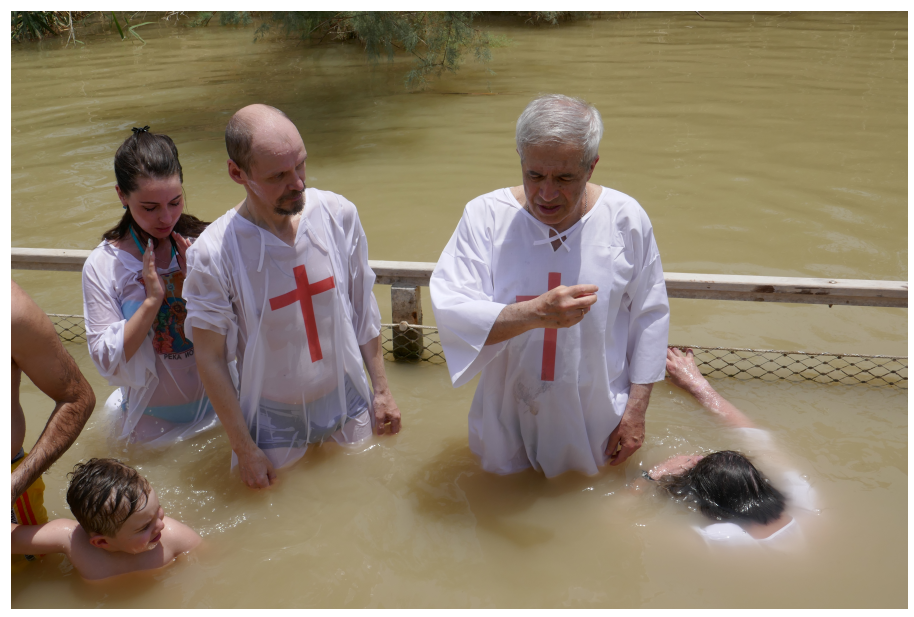

Figure 5. Alexandr Myllari and Vladimir Gerdt. Jordan river. 2017

\section{Dedication}

Professor Gerdt made numerous contributions to the fields of symbolic computation, differential algebra, and applications in physics. He was an excellent scientist and a kind-hearted and considerate man. Thank you very much, Vladimir!

\section{List of the published works of Vladimir Gerdt}

\subsection{Articles}

1. (with V. E. Aleinikov and M. M. Komochkov) Neutron Spectra Outside the Proton Accelerator Shielding, Neutron Monitoring for Radiation Protection Purposes, vol. I, IAEA, Vienna, 1973, 31-46.

2. (with V.A. Meshcheryakov and V.I. Zhuravlev) N-Scattering S Waves and the Value of the $\sigma$ commutator in the Static Model, Sov. J. Nucl. Phys., 20 (4), 1975, 405-407 (Yad. Fiz. 20, 4, 1974, 756-761, in Russian).

3. (with V.E. Aleinikov and M. M. Komochkov) Neutron Energy Spectra Outside the Shielding of High Energy Proton Accelerators, Proceedings of All-Union Meeting on Accelerators of Charged Particles, vol. II, Moscow: Nauka Publishers, 1975, 240-242. 
4. (with V.A. Meshcheryakov) Local Form of the Solution of the Chew-Low Equations, Teor. Mat. Fiz., 24, 2, 1975, 155-163.

5. (with V.E. Aleinikov and G. N. Timoshenko) Measurement of the Spectra of High Energy Protons from the Shielding of $680 \mathrm{Mev}$ Synchrocyclotron, Sov. Atomic Energy, 41, 5, 1976, 332-334.

6. (with V.I. Inozemtsev and V.A. Meshcheryakov) Uniformization of the Forward-Scattering Amplitude at High Energy, Lettere al Nuovo Cimento, $15,1976,321-328$.

7. (with V.E. Aleinikov and M. M. Komochkov) Some Regularities in Formation of the Neutron Spectra Outside the Shielding of Proton Accelerators, Sov. Atomic Energy, 42, 4, 1977, 305.

8. (with V.A. Meshcheryakov) Uniformization of the Forward Scattering Amplitude in the Quark Model, in Processes of Multiple Production and Inclusive Reactions at High Energy, Institute of High Energy Physics, Serpukhov, 1977, 333-340.

9. On Application of Computer Algebra Systems for Computation of Feynman Integrals, in Proceedings of International Meeting on Programming and Mathematical Methods for Solving the Physical Problems, Dubna, September 20-23, 1977, JINR D10,11-11264, Dubna, 1978, 166-174

10. Analytical Computation of the Invariant Curve of the Chew-Low Equations, U.S.S.R. Comput. Maths. Math. Phys, 19, 6, 1979, 257-266 (Zh. Vychisl. Mat. \& Mat. Fiz., 19, 6, 1979, 1602-1608, in Russian).

11. Local Construction of General Solution of the Chew-Low Equation by Computer, in Proceedings of International Conference on Systems and Techniques of Analytical Computing and Their Applications to Theoretical Physics, Dubna, September 18-21, 1979, JINR D11-80-13, 1980, 159-169.

12. (with O. V. Tarasov and D. V. Shirkov) Analytic Calculations on Digital Computers for Applications in Physics and Mathematics, Sov. Phys. Usp., 23 (1), 1980, 59-77 (Usp. Fiz. Nauk, 130, 1980, 113-147, in Russian).

13. (with A. Karimkhodzhaev and R. N. Faustov) Hadronic Vacuum Polarization and Test of Quantum Electrodynamics at Low Energies, in Problems in Theory of Gravity and Elementary Particles, K. P. Staniukovich (Ed.), Moscow: Atomizdat Publishers, 1980, 172-181.

14. Analytical Calculations in High Energy Physics by Computer, Computer Physics Communications, 20, 1980, 85-90.

15. Global Structure of the General Solution of the Chew-Low Equations, Sov. Theor. Math. Phys., 48, 3, 1982, 790-796 (Teor. Mat. Fiz., 48, 3, 1981, 346-355, in Russian).

16. Global Structure of the General Solution of the Chew-Low Equations, Sov. Theor. Math. Phys. 48, 3, 1982, 790-796 (Teor. Mat. Fiz., 48, 3, 1981, 346-355).

17. (with A. Yu. Zharkov) Solution of Chew-Low Equations in the Quadratic Approximation, Sov. Theor. Math. Phys., 52, 3, 1983, 868-874 (Teor. Mat. Fiz., 52, 3, 1982, 384-392, in Russian).

18. (with A. B. Shvachka and A. Yu. Zharkov) Investigation of Nonlinear Evolution Equations Using Analytical Calculation Systems, in Proceedings of the Second International Conference on Systems and Techniques of Analytical Computing and Their Applications in Theoretical Physics, Dubna, September 21-23, 1982, JINR D11-83-511, Dubna, 1983, 114-119. 
19. (with A. Yu. Zharkov) A REDUCE Package for Solving of Ordinary Differential Equations, in Proceedings of the Second International Conference on Systems and Techniques of Analytical Computing and Their Applications in Theoretical Physics, Dubna, September 21-23, 1982, JINR D11-83-511, Dubna, 1983, 171-177.

20. (with A. Yu. Zharkov) Iterative Method of Construction of General Solution of the Chew-Low Equation, in Proceedings of the Second International Conference on Systems and Techniques of Analytical Computing and Their Applications in Theoretical Physics, Dubna, September 21-23, 1982, JINR D11-83-511, Dubna, 1983, 232-241.

21. (with A. P. Kryukov, A. Ya. Rodionov and A. Yu. Zharkov) An Algorithm of Elementary Fraction Decomposition of Rational Functions and Its Implementation in System REDUCE, in Proceedings of the Second International Conference on Systems and Techniques of Analytical Computing and Their Applications in Theoretical Physics, Dubna, September 21-23, 1982, JINR D11-83-511, Dubna, 1983, 178-182.

22. (with O. V. Tarasov) Analytical Computations by Computer and its Application to High Energy Physics, in Proceedings of the XV International School on High Energy Physics for Young Scientists, Dubna, November 23 December 2, 1982, JINR D2-4-83-179, Dubna, 1983, 481-504.

23. (with V.K. Mitrjushkin) Phase Transitions in the Euclidean and Hamiltonian Approaches to Lattice Gauge Theories at a Finite Temperature, JETP Lett., 37, 8, 1983, 474-478 (Pis'ma Zh. Eksp. Teor. Fiz. 37, 8, 1983, 400-403, in Russian).

24. (with A. Yu. Zharkov) Cubic Approximation and Local Limitations on the Functional Arbitrariness in the General Solution of the Chew-Low Equations, Sov. Theor. Math. Phys., 52, 3, 1983, 626-639 (Teor. Mat. Fiz., 55, 3, 1983, 469-474, in Russian).

25. (with A. S. Ilchev and V.K. Mitrjushkin) Phase Transitions in Abelian Higgs Models on a Lattice, Sov. J. Nucl. Phys., 40, (4), 1985, 698-702 (Yad. Fiz., 40, 1984, 1097-1104, in Russian).

26. (with A. Yu. Zharkov) Methods of Investigating and Solving Differential Equations by Means of Algebraic Computation, in Systems for Analytical Transformations in Mechanics, Gorky, 1984, 16-19.

27. (with A. B. Shvachka and A. Yu. Zharkov) FORMINT - a Program for the Classification of Integrable Nonlinear Evolution Equations, Computer Physics Communications, 34, 1985, 303-311.

28. (with A.S. Ilchev, V.K. Mitrjushkin and A. M. Zadorozhny) $S U(2)$ Lattice Gauge-Higgs Model, Zeitschrift für Physik C - Particles and Fields, 29, 1985, 363-369.

29. (with A. B. Shvachka and A. Yu. Zharkov) Computer Algebra Application for Classification of Integrable Non-Linear Evolution Equations, Journal of Symbolic Computation, 1, 1, 1985, 101-107.

30. (with A. Yu. Zharkov) On Computer Algebra Application to Classification of Integrable Systems of Nonlinear Evolution Equations, in Proceedings of the Third International Conference on Computer Algebra and its Applications to Theoretical Physics, Dubna, September 17-20, 1985, JINR D11-85-791, Dubna, 1985, 225-330.

31. (with N. A. Kostov, R. P. Raychev and R. P. Roussev) Algebraic Models in Nuclear Physics and Group Theory Computation with Using of Computer 
Algebra Systems, in Proceedings of the Third International Conference on Computer Algebra and its Applications to Theoretical Physics, Dubna, September 17-20, 1985, JINR D11-85-791, Dubna, 1985, 376-381.

32. (with A. S. Ilchev, V. K. Mitrjushkin, I. K. Sobolev and A. M. Zadorozhny) Phase Structure of the $S U(2)$ Lattice Gauge Higgs Theory, Nuclear Physics, B265 [FS15], 1986, 145-160.

33. (with D. Yu. Grigor'ev) Computer Algebra Algorithms, Systems and Applications, in Computer Algebra. Symbolic and Algebraic Computation, Moscow: Mir Publishers, 1986, 373-383.

34. (with V.K. Mitrjushkin and A. M. Zadorozhny) The Phase Structure of the $S U(3)$ Lattice Gauge-Higgs Model, Physics Letters, 172B, 1, 1986, $65-70$.

35. (with A.S. Ilchev and V.K. Mitrjushkin) Lattice U(1) Higgs-Gauge Theory, Sov. J. Nucl. Phys., 43 (3), 1986, 468-473 (Yad. Fiz., 43, 1986, 736-746, in Russian).

36. (with O. V. Tarasov and D. V. Shirkov) Analytical Computations by Computer in Theoretical Physics, in Problems of Cybernetics. Computer Application to Quantum Field Theory, Moscow, 1987, 146-154.

37. (with V. K. Mitrjushkin and A. M. Zadorozhny) Phase Structure of GaugeHiggs Lattice Theory, Proceedings of International Seminar "Quarks-86", Tbilisi, April 15-17, 1986, Moscow, 1987, 300-307.

38. (with A. A. Bogoliubskaya and O. V. Tarasov) On Forming of Libraries for Computer Algebra Systems SCHOONSCHIP and REDUCE, in Applied Packages. Analytical Transformations, Moscow: Nauka Publishers, 1988, 83-90.

39. (with R. N. Fedorova, N. N. Govorun and V.P. Shirikov) Software for Analytical Computation, in Modern Problems of Applied Mathematics and Mathematical Physics, Moscow: Nauka Publishers, 1988, 150-160.

40. (with D. V. Shirkov) New Trends in Computer Science. Computer Algebra, in Computers in Modern Science, Moscow: Nauka Publishers, 1988, $35-48$.

41. (with O. V. Tarasov and D. V. Shirkov) Computer Algebra Application to High Energy Physics, in Proceedings of International School on the Problems of Use of Computers in Physical Research, Dubna, 28 November 3 December, 1988, JINR D10-89-70, Dubna, 1989, 134-178.

42. (with R. N. Fedorova, N.N. Govorun and V.P. Shirikov) Computer Algebra in Physical Research of Joint Institute for Nuclear Research, in EUROCAL'87, Lecture Notes in Computer Science, 378, Springer-Verlag, 1989, 1-10.

43. (with A. B. Shabat, S. I. Svinolupov and A. Yu. Zharkov) Computer Algebra Application to Investigating Integrability of Nonlinear Evolution Systems, in EUROCAL'8\%, Lecture Notes in Computer Science, 378, Springer-Verlag, 1989, 81-92.

44. (with A. Yu. Zharkov) Computer Classification of Integrable Seventh Order MKdV-Like Equations, in EUROCAL'87, Lecture Notes in Computer Science, 378, Springer-Verlag, 1989, 93-94.

45. (with N. A. Kostov and Z. T. Kostova) Computer Algebra and Computation of Puiseux Expansions of Algebraic Functions, in EUROCAL'87, Lecture Notes in Computer Science, 378, Springer-Verlag, 1989, 206-207. 
46. (with N. A. Kostov) Computer Algebra in the Theory of Ordinary Differential Equations of Halphen Type, in: Computer and Mathematics, E. Kaltofen and S. M. Watt (Eds.), New York: Springer-Verlag, 1989, $279-288$.

47. (with N. A. Kostov and A. Y. Spasov) Investigation of Four Wave Interaction in 4 Theory Using Computer Algebra, in: Solitons and Applications, V. G. Makhankov, V.K. Fedyanin and O. K. Pashaev (Eds.), Singapore: World Scientific Publishing Co., 1990, 114-119.

48. (with N. A. Kostov and A. Yu. Zharkov) Nonlinear Evolution Equations and Solving Algebraic Systems: the Importance of Computer Algebra, in Solitons and Applications, V. G. Makankov, V. K. Fedyanin and O. K. Pashaev (Eds.), Singapore: World Scientific Publishing Co., 1990, 120-128.

49. (with A. Yu. Zharkov) Computer Classification of Integrable Coupled KdV-Like Systems, Journal of Symbolic Computation, 10, 1990, 203-207.

50. (with A. Yu. Zharkov) Computer Generation of Necessary Integrability Conditions for Polynomial-Nonlinear Evolution Systems, in Proceedings of "ISSAC'90", International Symposium on Symbolic and Algebraic Computation, ACM Press, Addison-Wesley Publishing Company, 1990, 250-254.

51. (with N. V. Khutornoy and A. Yu. Zharkov) Lie-Bäcklund Symmetries of Coupled Nonlinear Schrödinger Equations, in Proceedings of "ISSAC'91", International Symposium on Symbolic and Algebraic Computation, ACM Press, Addison-Wesley Publ. Company, 1991, 313-314

52. Integrability of Polynomial-Nonlinear Evolution Equations and Computer Algebra, in: Nonlinear Evolution Equations and Dynamical Systems, V.G. Makhankov and O.K. Pashaev (Eds.), Springer-Verlag, Berlin, 1991, 121-123.

53. (with V. E. Kovtun and V. N. Robuk) Genetic Codes of Lie Algebras and Nonlinear Evolution Equations, in Nonlinear Evolution Equations and Dynamical Systems, V. G. Makhankov and O. K. Pashaev (Eds.), Berlin: Springer-Verlag, 1991, 124-126.

54. (with I. R. Akselrod, V.E. Kovtun and V. N. Robuk) Construction of a Lie Algebra by a Subset of Generators and Commutation Relations, in Computer Algebra in Physical Research, D. V. Shirkov, V. A. Rostovtsev and V.P. Gerdt (Eds.), Singapore: World Scientific Publ. Co., 1991, 306-312.

55. (with N. V. Khutornoy and A. Yu. Zharkov) Solving Algebraic Systems Which Arise as Necessary Integrability Conditions for PolynomialNonlinear Evolution Equations, in Computer Algebra in Physical Research, D. V. Shirkov, V. A. Rostovtsev and V.P. Gerdt (Eds.), World Scientific Publ. Co., Singapore, 1991, 321-328.

56. (with L. M. Berkovich, Z.T. Kostova and M. L. Nechaevsky) Integration of Some Classes of Linear Ordinary Differential Equations, in Computer Algebra in Physical Research, D. V. Shirkov, V. A. Rostovtsev and V. P. Gerdt (Eds.), Singapore: World Scientific Publ. Co., 1991, 350-356.

57. (with O. V. Tarasov and D. V. Shirkov) Symbolic and Formula Processing in HEP, in Computing in High Energy Physics'91, Y. Watase and F. Abe (Eds.), Tokyo: Universal Academy Press Inc., 1991, 373-382.

58. Computer Algebra Tools for Higher Symmetry Analysis of Nonlinear Evolution Equations, in Programming Environments for High-Level Scientific 
Problem Solving, P. W. Gaffney and E. N. Houstis (Eds.), North-Holland, 1992, 107-115.

59. (with N. V. Khutornoy and A. Yu. Zharkov) Gröbner Basis Technique, Homogeneity and Solving Polynomial Equations, in Proceedings of the 1992 International Workshop on Mathematics Mechanization, Beijing, China, July 16-18, 1992, Wu Wen-Tsün and Cheng Min-De (Eds.), Beijing, China: International Academic Publishers, 1992, 38-51.

60. (with L. M. Berkovich, Z. T. Kostova and M. L. Nechaevsky) Second Order Reducible Linear Differential Equations, in Applied Packages. Software for Mathematical Simulation, Moscow: Nauka Publishers, 1992, 9-24.

61. Computer Algebra, Symmetry Analysis and Integrability of Nonlinear Evolution Equations, in Physics Computing'92, R. A. de Groot and J. Nadrchal (Eds.), Singapore: World Scientific Publ. Co., 1993, 52-59; International Journal of Modern Physics $C$, vol. 4, no. 2, 1993, 279-286.

62. (with N. V. Khutornoy and A. Yu. Zharkov) ASYS: A Computer Algebra Package for Analysis of Systems of Nonlinear Algebraic Equations, Russian Journal "Programming and Computer Software", no. 2, 1993, 69-75.

63. (with W. Lassner) Isomorphism Verification for Complex and Real Lie Algebras by Gröbner Basis Technique, in Modern Group Analysis: Advanced Analytical and Computational Methods in Mathematical Physics, N. H. Ibragimov et al. (Eds.), Amsterdam: Kluwer Academic Publishers, 1993, 245-254.

64. (with N. V. Khutornoy and A. Yu. Zharkov) ASYS2: a New Version of Computer Algebra Package ASYS for Analysis and Simplification of Polynomial Systems, in Proceedings of the Rhein Workshop on Computer Algebra Karlsruhe, Germany, March 22-24, 1994, J. Calmet (Ed.), Institute of Algorithms and Cognitive Systems, University of Karlsruhe, 1994, $162-178$.

65. (with N. Khutornoy and W. Lassner) Computer Detecting of Lie Algebra Isomorphisms, in Programming and Mathematical Technique in Physics, Yu. Yu. Lobanov and E. P. Zhidkov (Eds.), Singapore: World Scientific, 1994, 108-113.

66. (with N. V. Khutornoy and A. Yu. Zharkov) Implementation of ZeroDimensional Gröbner Bases Transformation from One Order into Another, in Proceedings of the International Workshop on New Computer Technologies in Control Systems, Perslavl-Zalessky, Russia, July 11-15, 1994, Program Systems Institute, Pereslavl-Zalessky, 1994, 36-43.

67. (with V. V. Kornyak) Lie Algebras and Lie Superalgebras Defined by a Finite Number of Relations: Computer Analysis, Journal of Nonlinear Mathematical Physics, vol. 2, no. 3-4, 1995, 367-373.

68. (with V. V. Kornyak) Computer Analysis of Finitely Presented Lie Superalgebras, in New Computing Techniques in Physics Research IV, B. Denby and D. Perret-Gallix (Eds.), Singapore: World Scientific, 1996, 289-294.

69. Homogeneity of Integrability Conditions for Multiparametric Families of Polynomial-Nonlinear Evolution Equations, Mathematics and Computers in Simulation, 42, 1996, 399-408.

70. (with V. V. Kornyak) Construction of Finitely Presented Lie Algebras and Superalgebras, Journal of Symbolic Computation, 21, 1996, 337-349. 
71. (with V. F. Edneral, D. V. Shirkov and N. N. Vasiliev) Computer Algebra as Applied to Science and Engineering, Russian Journal "Programming and Computer Software", 22, 6, 1996, 296-306.

72. (with V.N. Robuk and V.M. Severyanov) On Construction of Finitely Presented Lie Algebras, Russian Journal of Computational Mathematics and Mathematical Physics, 36, 11, 1996, 1493-1505 (Zh. Vychisl. Mat. \& Mat. Fiz., in Russian).

73. (with V. V. Kornyak) An Implementation in $\mathrm{C}$ of an Algorithm for Construction of Finitely Presented Lie Superalgebras, Computer Science Journal of Moldova, vol. 4, no. 3, 1996, 399-427.

74. (with V. V. Kornyak) A Program for Constructing the Complete Set of Relations, Basis Elements and Their Commutator Table for Finitely Presented Lie Algebras and Superalgebras, Russian Journal "Programming and Computer Software", 23, 1997, 164-172.

75. Gröbner Bases and Involutive Methods for Algebraic and Differential Equations, Mathematics and Computers in Modelling, vol. 25, no. 8-9, 1997, 75-90.

76. (with V. V. Kornyak) A Program for Constructing Finitely Presented Lie Algebras and Superalgebras, Nuclear Instruments 8 Methods in Physics Research A, 389, 1997, 370-373.

77. (with V.V. Kornyak) An Algorithm for Analysis of the Structure of Finitely Presented Lie Algebras and Superalgebras, Discrete Mathematics and Theoretical Computer Science, 1, 1997, 217-228.

78. (with Yu. A. Blinkov) Involutive Bases of Polynomial Ideals, Mathematics and Computers in Simulation, 45, 1998, 519-542.

79. (with Yu. A. Blinkov) Minimal Involutive Bases, Mathematics and Computers in Simulation, 45, 1998, 543-560.

80. (with M. Berth and G. Czichowski) Involutive Divisions in Mathematica: Implementation and Some Applications, in Proceedings of the 6th Rhein Workshop on Computer Algebra, Sankt-Augustin, Germany, March 31 April 3, 1998, J. Calmet (Ed.), Institute for Algorithms and Scientific Computing, GMD-SCAI, Sankt-Augustin, 1998, 74-91.

81. (with M. Berth and G. Czichowski) Completion of Monomial Sets to Involution with Mathematica, International Conference "Computer Algebra in Scientific Computing", April 20-24, St. Petersburg, Russia, Extended Abstracts, Euler International Mathematical Institute, St. Petersburg, 1998, 58-63.

82. (with Yu. A. Blinkov) Involutive Monomial Divisions, Russian Journal "Programming and Computer Software", vol. 24, no. 6, 1998, 283-285.

83. Completion of Linear Differential Systems to Involution, in Computer Algebra in Scientific Computing, CASC'99, V. G. Ganzha, E. W. Mayr and E. V. Vorozhtsov (Eds.), Berlin: Springer-Verlag, 1999, 0115-0137.

84. (with S.A. Gogilidze) Constrained Hamiltonian Systems and Gröbner Bases, in Computer Algebra in Scientific Computing, CASC'99, V. G. Ganzha, E. W. Mayr and E. V. Vorozhtsov (Eds.), Berlin: SpringerVerlag, 1999, 138-146.

85. (with V. V. Kornyak, M. Berth and G. Czichowski) Construction of Involutive Monomial Sets for Different Involutive Divisions, in Computer Algebra in Scientific Computing, CASC'99, V. G. Ganzha, E. W. Mayr and E. V. Vorozhtsov (Eds.), Berlin: Springer-Verlag, 1999, 147-157. 
86. (with M. G. Dmitriev and M. V. Nesterova) Polynomial-Nonlinear Boundary Problems with Inexactly Known Boundary Conditions and their Approximate Solving by Means of Gröbner Bases, Russian Journal "Fundamental and Applied Mathematics", 5, 1999, 675-686.

87. Computer Algebra and Constrained Dynamics, in Problems of Modern Physics, A. N. Sisakian and D. I. Trubetskov (Eds.), JINR D2-99-263, $2000,164-171$.

88. On the Relation Between Pommaret and Janet Bases, in Computer Algebra in Scientific Computing, CASC 2000, V. G. Ganzha, E. W. Mayr, E. V. Vorozhtsov (Eds.), Berlin: Springer-Verlag, 2000, 164-171.

89. (with Yu. A. Blinkov and D. A. Yanovich) Fast Search for the Janet Divisor, Russian Journal "Programming and Computer Software", vol. 27, no. $1,2001,22-24$.

90. Involutivity Applied to Differential Equations, in Proceedings of International Conference "Differential Equations and Computer Algebra Systems", Brest, Belarus, September 19-22, 2000, Brest State University, 2001, $10-15$.

91. (with M. Berth) Computation of Involutive Bases with Mathematica, in Proceedings of the Third International Workshop on Mathematica System in Teaching and Research, Sieldce, Poland, September 5-7, 2001, Institute of Mathematics \& Physics, University of Podlasie, 2001, 29-34.

92. (with Yu. A. Blinkov and D. A. Yanovich) Computation of Janet Bases. I. Monomial Bases, in Computer Algebra in Scientific Computing, CASC 2001, V. G. Ganzha, E. W. Mayr, E. V. Vorozhtsov (Eds.), Berlin: Springer-Verlag, 2001, 233-247.

93. (with Yu. A. Blinkov and D. A. Yanovich) Computation of Janet Bases. II. Polynomial Bases, in Computer Algebra in Scientific Computing, CASC 2001, V. G. Ganzha, E. W. Mayr, E. V. Vorozhtsov (Eds.), Berlin: Springer-Verlag, 2001, 249-263.

94. On an Algorithmic Optimization in Computation of Involutive Bases, Russian Journal "Programming and Computer Software", vol. 28, no. 2, 2002, 62-65.

95. Involutive Division Technique: Some Generalizations and Optimizations, Journal of Mathematical Sciences, 108 (6), 2002, 1034-1051.

96. (with Yu. A. Blinkov) Janet Bases of Toric Ideals, in Computer Algebra and its Application to Physics, CAAP-2001, V.P.Gerdt (Ed.), JINR E5,112001-279, Dubna, 2002, 71-82; Proceedings of the 8th Rhine Workshop on Computer Algebra, Mannheim, Germany, March 21-22, 2002, H. Kredel, W. K. Seiler (Eds.), University of Mannheim, 2002, 125-135.

97. (with A. M. Khvedelidze and D. M. Mladenov) Analysis of Constraints in Light-cone Version of SU(2) Yang-Mills Mechanics, in Computer Algebra and its Application to Physics, CAAP-2001, V.P. Gerdt (Ed.), JINR E5,11-2001-279, Dubna, 2002, 83-92.

98. (with D. A. Yanovich) Parallelism in Computing Janet Bases, in Computer Algebra and its Application to Physics, CAAP-2001, V.P. Gerdt (Ed.), JINR E5,11-2001-279, Dubna, 2002, 93-103; Proceedings of the Workshop on Under- and Overdetermined Systems of Algebraic or Differential Equations, Karlsruhe, March 18-19, 2002, J. Calmet, M. Hausdorf, W. M. Seiler (Eds.), Institute of Algorithms and Cognitive Systems, University of Karlsruhe, 2002, 47-56. 
99. (with G. Carra'Ferro) Extended Characteristic Sets of Finitely Generated Differential Ideals, in Computer Algebra in Scientific Computing, CASC 2002, V. G. Ganzha, E. W. Mayr, E. V. Vorozhtsov (Eds.). Institute of Informatics, Technical University of Munich, Garching, 2002, 29-36.

100. (with D. A. Yanovich) Implementation of the FGLM Algorithm and Finding Roots of Polynomial Involutive Systems, Programming and Computer Software, vol. 29, no. 2, 2003, 72-74.

101. (with G. Carra'Ferro) Improved Kolchin-Ritt Algorithm, Programming and Computer Software, vol 29, No. 2, 2003, 83-87.

102. (with M. Znojil and D. A. Yanovich) New exact solutions for polynomial oscillators in large dimensions, Journal of Physics A: Mathematical and General, 36, 2003, 6531-6545.

103. (with Yu. A. Blinkov, C. F. Cid, W. Plesken and D. Robertz) The Maple Package "Janet": I. Polynomial Systems, in Computer Algebra in Scientific Computing, CASC 2003, V. G. Ganzha, E. W. Mayr, E. V. Vorozhtsov (Eds.). Institute of Informatics, Technical University of Munich, Garching, 2003, 31-40.

104. (with Yu. A. Blinkov, C.F. Cid, W. Plesken and D. Robertz) The Maple Package "Janet": II. Linear Partial Differential Equations, in Computer Algebra in Scientific Computing, CASC 2003, V. G. Ganzha, E. W. Mayr, E. V. Vorozhtsov (Eds.), Institute of Informatics, Technical University of Munich, Garching, 2003, 41-54.

105. (with M. Znojil and D. A. Yanovich) On Exact Solvability of Anharmonic Oscillators in Large Dimensions, in Computer Algebra in Scientific Computing, CASC 2003, V. G. Ganzha, E. W. Mayr, E. V. Vorozhtsov (Eds.), Institute of Informatics, Technical University of Munich, Garching, 2003, $143-162$.

106. (with D. A. Yanovich) Parallel Computation of Involutive and Gröbner Bases, in Computer Algebra in Scientific Computing, CASC 2004, V. G. Ganzha, E. W. Mayr, E. V. Vorozhtsov (Eds.), Institute of Informatics, Technical University of Munich, Garching, 2004, 185-194.

107. Gröbner Bases in Perturbative Calculations. Nuclear Physics B (Proc. Suppl.), 135, 2004, 232-237.

108. (with D. A. Yanovich) Parallel Computation of Janet and Gröbner Bases over Rational Numbers. Programming and Computer Software, vol. 31, no. 2, 2005, 73-80.

109. Involutive Algorithms for Computing Gröbner Bases, in Computational Commutative and Non-Commutative Algebraic Geometry, S. Cojocaru, G. Pfister and V. Ufnarovski (Eds.), NATO Science Series, IOS Press, 2005, 199-225.

110. (with Yu. A. Blinkov) Janet-like Monomial Division, in Computer Algebra in Scientific Computing, CASC 2005, V. G. Ganzha, E. W. Mayr, E. V. Vorozhtsov (Eds.), LNCS, 3781, Berlin: Springer-Verlag, 2005, $174-183$.

111. (with Yu. A. Blinkov) Janet-like Gröbner Bases, in Computer Algebra in Scientific Computing, CASC 2005, V. G. Ganzha, E. W. Mayr, E. V. Vorozhtsov (Eds.), LNCS, 3781, Berlin: Springer-Verlag, 2005, $184-195$.

112. (with A. Gusev, M. Kaschiev, V. Rostovtsev, V. Samoylov, T. Tupikova, Y. Uwano and S. Vinitsky) Symbolic-Numerical Algorithm for Solving 
the Time-Dependent Shrödinger Equation by Split-Operator Method, in Computer Algebra in Scientific Computing, CASC 2005, V. G. Ganzha, E. W. Mayr, E. V. Vorozhtsov (Eds.), LNCS, 3781, Berlin: SpringerVerlag, 2005, 244-258.

113. (with D. A. Yanovich) Experimental Analysis of Involutive Criteria, in Algorithmic Algebra and Logic, A. Dolzmann, A. Seidl and T. Sturm (Eds.), BOD Norderstedt, Germany, 105-109.

114. (with D. Robertz) Computation of Gröbner Bases for Systems of Linear Difference Equations, Computeralgebra, Rundbrief, Nr. 37, GI_DMV_GAMM, 2005, 8-13.

115. (with Yu. A. Blinkov) On Computing Janet Bases for Degree Compatible Orderings, in Proceedings of the 10th Rhine Workshop on Computer Algebra Basel, Switzerland, March 16-17, 2006, J. Draisma and H. Kraft (Eds.), University of Basel, 2006, 107-117.

116. On Computation of Gröbner Bases for Linear Difference Systems. Nuclear Instruments and Methods in Physics Research, 559 (1), 2006, 211-214.

117. (with D. Robertz) A Maple Package for Computing Gröbner Bases for Linear Recurrence Relations. Nuclear Instruments and Methods in Physics Research, 559 (1), 2006, 215-219.

118. (with V.M. Severyanov) A Software Package to Construct Polynomial Sets over Z2 for Determining the Output of Quantum Computation. Nuclear Instruments and Methods in Physics Research A, 559 (1), 2006, 215-219, 260-264.

119. (with Yu. A. Blinkov and V. V. Mozzhilkin) Gröbner Bases and Generation of Difference Schemes for Partial Differential Equations, Symmetry, Integrability and Geometry: Methods and Applications (SIGMA), 2, 2006, $051,26 \mathrm{p}$.

120. (with S.I. Vinitsky, A. A. Gusev, M. S. Kaschiev, V. A. Rostovtsev, V. N. Samoylov, T. V. Tupikova, Y. Uwano) Symbolic algorithm for factorization of evolution operator for time-dependent Schrödinger equation, Programming and Computer Software, vol. 32, no. 2, 2006, 103-113.

121. (with D. A. Yanovich) Effectiveness of Involutive Criteria in Computation of Polynomial Janet Bases, Programming and Computer Software, vol. 32, no. 3, 2006, 134-138.

122. (with A. Gusev, M. Kaschiev, V. Rostovtsev, V. Samoylov, T. Tupikova, S. Vinitsky) A Symbolic-Numerical Algorithm for Solving the Eigenvalue Problem for a Hydrogen Atom, in Computer Algebra in Scientific Computing, CASC 2006, V. G. Ganzha, E. W. Mayr, E. V. Vorozhtsov (Eds.), LNCS, 4194, Berlin: Springer-Verlag, 2006, 205-218.

123. (with A. Khvedelidze, Yu. Palii) Towards an Algorithmization of the Dirac Constraints Formalism, in Global Integrability of Field Theories, J. Calmet, W. M. Seiler, R. W. Tucker (Eds.), Cocroft Institute, Daresbury (UK), 2006.

124. (with V. M. Severyanov) An Algorithm for Constructing Polynomial Systems Whose Solution Space Characterizes Quantum Circuits, in Quantum Informatics 2005, Yu. I. Ozhigov (Ed.), Proceedings of SPIE, vol., 6264, 626401, 2006. 
125. (with R. Horan, A. Khvedelidze, M. Lavelle, D. McMullan, Yu. Palii) On the Hamiltonian reduction of geodesic motion on $\mathrm{SU}(3)$ to $\mathrm{SU}(3) / \mathrm{SU}(2)$, Journal of Mathematical Physics, vol. 47, no. 10, 2006, 112902.

126. Involutive methods applied to algebraic and differential equations, in Constructive algebra and Systems Theory, B. Hanzon, M. Hazewinkel (Eds.), Amsterdam: Royal Netherland Academy of Arts and Sciences, 2006, 245-250.

127. (with A. M. Khvedelidze and D. M. Mladenov) On application of involutivity analysis of differential equations to constrained dynamical systems, Symmetries and Integrable Systems, Selected Papers of the Seminar, 2000-2005, A. N. Sissakian (Ed.), vol. 1, Dubna, JINR, 132-150, 2006.

128. (with R. Kragler, A. N. Prokopenya) Mathematica Package for Construction of Circuit Matrices in Quantum Computation, in Computer Algebra Systems in Teaching and Research, CASTR 2007, University of Podlasie, Scieldce, Poland, 2007, 135-144.

129. (with V. M. Severyanov) C\# Package for Assembling Quantum Circuits and Generating Associated Polynomial Sets, Physics of Particles and Nuclei Letters, vol. 4, no. 2, 2007, 225-230.

130. (with A. Gusev, M. Kaschiev, V. Rostovtsev, V. Samoylov, T. Tupikova, Y. Uwano, S. Vinitsky) On Symbolic-Numerical Representation of Evolution Operator for Finite-Dimensional Quantum Systems, Particles and Nuclei Letters, vol. 4, no. 2, 2007, 253-259.

131. (with O. Chuluunbaatar, A. Gusev, M. Kaschiev, V. Rostovtsev, V. Samoylov, T. Tupikova, S. Vinitsky) Symbolic-Numeric Algorithm for Computing Matrix Elements of Parametric Eigenvalue Problem, Programming and Computer Software, vol. 33, no. 2, 105-116, 2007.

132. (with Yu. A. Blinkov) On Selection Strategy for Nonmultiplicative Prolongations at Construction of Janet Bases, Programming and Computer Software, vol. 33, no. 3, 147-153, 2007.

133. (with O. Chuluunbaatar, A. Gusev, M. Kaschiev, V. Rostovtsev, V. Samoylov, T. Tupikova, S. Vinitsky) Symbolic-Numerical Algorithm for Solving the Eigenvalue Problem for Hydrogen Atom in the Magnetic Field: Cylindrical Coordinates, in Computer Algebra in Scientific Computing, CASC 200\%, V. G. Ganzha, E. W. Mayr, E. V. Vorozhtsov (Eds.), LNCS, 4770, Berlin: Springer-Verlag, 2007, 118-133.

134. (with A. M. Khvedelidze, Yu. G. Palii) Deducing the constraints in the light-cone SU(3) Yang-Mills mechanics via Gröbner bases, in Computer Algebra in Scientific Computing, CASC 200\%, V. G. Ganzha, E. W. Mayr, E. V. Vorozhtsov (Eds.), LNCS, 4770, Berlin: Springer-Verlag, 2007, $145-159$.

135. (with R. Kragler, A. N. Prokopenya) On computer algebra application to simulation of quantum computation. Models and Methods in Few-and Many-Body Systems, in Proceedings of the DST-UNISA-JINR symposium, University of South Africa, Pretoria, 2007, 219-232.

136. On Completion to Involution Based on Janet Division, Computer Algebra and Differential Equations, Acta Academiae Aboensis, Ser. B, vol. 67, no. 2, 2007, 1-11.

137. (with R. Kragler, A. N. Prokopenya) A Mathematica Package for Construction of Circuit Matrices in Quantum Computation, Computer Algebra 
and Differential Equations, Acta Academiae Aboensis, Ser. B, vol. 67, no. 2, 2007, 28-38.

138. (with M. V. Zinin) On computation of Gröbner bases over F2. Computer Algebra and Differential Equations, Acta Academiae Aboensis, Ser. B, vol. 67, no. 2, 2007, 59-68.

139. (with O. Chuluunbaatar, A. A. Gusev, V. A. Rostovtsev, T. V. Tupikova, S. I. Vinitsky, A. G. Abrashkevich, M. S. Kaschiev, V. V. Serov) POTHMF, a program to compute matrix elements of the coupled radial equations for a Hydrogen-like atom in a homogeneous magnetic field, Computer Algebra and Differential Equations, Acta Academiae Aboensis, Ser. B, vol. 67, no. 2, 2007, 69-78.

140. (with Yu. A. Blinkov) On computer algebra-aided stability analysis of difference schemes generated by means of Gröbner bases, Computer Algebra and Differential Equations, Acta Academiae Aboensis, Ser. B, vol. 67 , no. 2 , 2007, 168-177.

141. (with O. Chuluunbaatar, A. A. Gusev, S,I. Vinitsky, A. G. Abrashkevich, M. S. Kaschiev, V. V. Serov) POTHMF: A program for computing potential curves and matrix elements of the coupled adiabatic radial equations for a Hydrogen-like atom in a homogeneous magnetic field, Computer Physics Communications, 178, 2008, 301-330.

142. On Decomposition of Algebraic PDE Systems into Simple Subsystems, Acta Appl. Math., 101, 2008, 39-51.

143. Gröbner Bases Applied to Systems of Linear Difference Equations, Physics of Particles and Nuclei Letters, vol. 5, no. 3, 2008, 425-436.

144. (with A. M. Khvedelidze, Yu. G. Palii) Light-cone Yang-Mills mechanics: SU(2) vs. SU(3), Theoretical and Mathematical Physics, 155 (1), 2008, $557-566$.

145. (with Yu. A. Blinkov) Specialized Computer Algebra System GINV, Programming and Computer Software, vol. 34, no. 2, 2008, 112-123.

146. (with M. V. Zinin) Involutive Method for Computing Gröbner Bases over F2, Programming and Computer Software, vol. 34, no. 4, 2008, 191-203.

147. (with M. V. Zinin) A Pommaret Division Algorithm for Computing Gröbner Bases in Boolean Rings, Proceedings of ISSAC 2008, ACM Press, 95-102.

148. (with Nguyen Van Hieu, Nguyen Bich Ha, O. Chuluunbaatar, A. A. Gusev, Yu. G. Palii and Nguyen Van Hop) Analytical Asymptotic Expressions for the Green's Function of the Electron in a Single-Level Quantum Dot at the Kondo and the Fano Resonances, Journal of the Korean Physical Society, vol. 53, no. 6, 2008, 3645-3649.

149. (with M. V. Zinin) Role of Involutive Criteria in Computing Boolean Gröbner Bases, Programming and Computer Software, vol. 35, no. 2, 2009, 90-97.

150. (with M. Eliashvili, A. M. Khevedelidze) On precession of entangled spins in a strong laser field, Physics of Atomic Nuclei, vol. 72, no. 5, 2009, $1-8$.

151. (with Yu. A. Blinkov and M. V. Zinin) On computation of Boolean involutive bases, in Proceedings of the 2nd International conference "Polynomial Computer Algebra", POMI RAS, St. Petersburg, 2009, 17-24.

152. (with Yu. A. Blinkov) Involution and Difference Schemes for the NavierStokes Equations, in Computer Algebra in Scientific Computing, CASC 
2009, V. P. Gerdt, E. W. Mayr, E. V. Vorozhtsov (Eds.), LNCS, 5743, Berlin: Springer-Verlag, 2009, 94-105.

153. (with R. Kragler, A. N. Prokopenya) A Mathematica Package for Simulation of Quantum Computation, in Computer Algebra in Scientific Computing, CASC 2009, V.P. Gerdt, E. W. Mayr, E. V. Vorozhtsov (Eds.), LNCS, 5743, Berlin: Springer-Verlag, 2009, 106-117.

154. (with S. I. Vinitsky, O. Chuluunbaatar, A. A. Gusev, V. A. Rostovtsev) Symbolic-Numerical Algorithms for Solving Parabolic Quantum Well Problem with Hydrogen-Like Impurity, in Computer Algebra in Scientific Computing, CASC 2009, V. P.Gerdt, E. W. Mayr, E. V. Vorozhtsov (Eds.), LNCS, 5743, Berlin: Springer-Verlag, 2009, 334-349.

155. On Completion of Nonlinear Differential Systems to Involution, in Proceedings of the 4th World Conference on 21st Century Mathematics, A. D. R. Choudary (Ed.), Lahore, Pakistan, 2009, 79-87.

156. (with M. V. Zinin) An algorithmic approach to solving polynomial equations associated with quantum circuits, Physics of Particles and Nuclei Letters, vol. 6, no. 7, 2009, 521-525.

157. (with R. Kragler, A. N. Prokopenya) A Mathematica Program for Constructing Quantum Circuits and Computing Their Unitary Matrices, Physics of Particles and Nuclei Letters, vol. 6, no. 7, 2009, 526-529.

158. (with O. Chuluunbaatar, A. A. Gusev, M. S. Kaschiev, V. A. Rostovtsev, Y. Uwano, S. I. Vinitsky) Multi-Layer Evolution Schemes for the FiniteDimensional Quantum Systems in External Fields, Physics of Particles and Nuclei Letters, vol. 6, no. 7, 2009, 550-553.

159. Algebraically Simple Involutive PDEs and Cauchy Problem, Journal of Mathematical Sciences, vol. 168, no. 3, 2010, 362-367.

160. (with A. Khvedelidze and Yu. Palii) On the ring of local invariants for a pair of entangles qubits, Journal of Mathematical Sciences, vol. 168, no. 3, 2010, 368-378.

161. (with D. Stefanescu and S. Yevlakhov) Estimations of Positive Roots of Polynomials, Journal of Math ematical Sciences, vol. 168, no. 3, 2010, 468-474.

162. (with A. N. Prokopenya) On Some Algorithms of Computing Unitary Matrices for Quantum Circuits, Programming and Computer Software, vol. 36, no. 2, 2010, 111-116.

163. (with Yu. A. Blinkov and M. V. Zinin) On Computing Boolean Involutive Bases, Programming and Computer Software, vol. 36, no. 2, 2010, 117-123.

164. (with D. Robertz) Consistency of Finite Difference Approximations for Linear PDE Systems and its Algorithmic Verification, in Proceedings of ISSAC 2010, ACM Press, 2010, 53-59.

165. (with T. Bächler, M. Lange-Hegermann and D. Robertz) Thomas Decomposition of Algebraic and Differential Systems, in Computer Algebra in Scientific Computing, CASC 2010, V. P. Gerdt, W. Koepff, E. W. Mayr, E. V. Vorozhtsov (Eds.), LNCS, 6264, Berlin: Springer-Verlag, 2010, 31-54.

166. (with A. A. Gusev, O. Chuluunbaatar, V. A. Rostovtsev, S. I. Vinitsky, V.L. Derbov and V. V. Serov) Symbolic-Numeric Algorithms for Computer Analysis of Spheroidal Quantum Dot Models, in Computer Algebra in Scientific Computing, CASC 2010, V. P. Gerdt, W. Koepff, E. W. Mayr, 
E. V. Vorozhtsov (Eds.), LNCS, 6264, Berlin: Springer-Verlag, 2010, $106-122$.

167. (with A. N. Prokopenya) Simulation of the quantum algorithm for order finding with the QuantumCircuit package, Vestnik of Brest Technical University, Series Physics, Mathematics, Informatics, no. 5, 2010, 780-83, in Russian.

168. (with A. Khvedelidze and Yu. Palii) Constraints on $S U(2) \otimes S U(2)$ invariant polynomials for entangled qubit pair, Physics of Atomic Nucleii, vol. 74, no. 6, 2011, 893-900.

169. (with Yu. A. Blinkov) Involutive Division Generated by an Antigraded Monomial Ordering, in Computer Algebra in Scientific Computing, CASC 2011, V. P. Gerdt, W. Koepff, E. W. Mayr, E. V. Vorozhtsov (Eds.), LNCS, 6885, Berlin: Springer-Verlag, 2011, 158-174.

170. (with A. A. Gusev, S. I. Vinitsky, O. Chuluunbaatar and V. A. Rostovtsev) Symbolic-Numerical Algorithms to Solve the Quantum Tunneling Problem for a Coupled Pair of Ions, in Computer Algebra in Scientific Computing, CASC 2011, V. P. Gerdt, W. Koepff, E. W. Mayr, E. V. Vorozhtsov (Eds.), LNCS, 6885, Berlin: Springer-Verlag, 2011, 175-191.

171. (with A. Khvedelidze and Yu. Palii) Separability of Two-Qubit State in Terms of Local Invariants, Physics of Particles and Nuclei Letters, vol. 8, no. 5, 2011, 451-454.

172. (with A. Khvedelidze, D. Mladenov and Yu. Palii) SU(6) Casimir Invariants and $S U(2) \otimes S U(3)$ Scalars for a Mixed Qubit-Qutrit States, Journal of Mathematical Sciences, vol. 179, no. 6, 2011, 690-701.

173. Consistency Analysis of Finite Difference Approximations to PDE Systems, in Proceedings of MMCP 2011, July 3-8, 2011, Stara Lesna, High Tatra Mountains, Slovakia, G. Adam, J. Busa, M. Hnatic (Eds.), LNCS, 7175, Heidelberg: Springer, 2012, 28-42.

174. (with A. N. Prokopenya) The Circuit Model of Quantum Computation and its Simulation with Mathematica, in Proceedings of MMCP 2011, July 3-8, 2011, Stara Lesna, High Tatra Mountains, Slovakia, G. Adam, J. Busa, M. Hnatic (Eds.), LNCS, 7175, Heidelberg: Springer, 2012, 43-55.

175. (with T. Bächler, M. Lange-Hegermann and D. Robertz) Algorithmic Thomas Decomposition of Algebraic and Differential Systems, Journal of Symbolic Computation, 47 (10), 1233-1266, 2012.

176. (with A. Hashemi and B. M. Alizadeh) A Variant of Gerdt's Algorithm for Computing Involutive Bases, Bulletin of Peoples' Friendship University of Russia: Series Mathematics, Information Sciences, Physics, no. 2, 2012, 66-77.

177. (with A. Hashemi) Comprehensive Involutive Systems, in Computer Algebra in Scientific Computing, CASC 2012, V.P. Gerdt, W. Koepff, E. W. Mayr, E. V. Vorozhtsov (Eds.), LNCS, 7442, Berlin: SpringerVerlag, 2012, 98-116.

178. (with A. Gusev, S. Vinitsky, O. Chuluunbaatar, L. Le Hai, V. Rostovtsev) Symbolic-Numerical Algorithm for Calculations of High- $|\mathrm{m}|$ Rydberg States and Decay Rates, in Computer Algebra in Scientific Computing, CASC 2012, V. P. Gerdt, W. Koepff, E. W. Mayr, E. V. Vorozhtsov (Eds.), LNCS, 7442, Berlin: Springer-Verlag, 2012, 155-171. 
179. On investigation of finite difference approximations to partial differential equations systems, Electronic journal "System analysis in science and education", no. 2, 2012, in Russian. URL: http://www.sanse.ru/archive/24.

180. (with D. Robertz) Computation of Difference Gröbner Bases, Computer Science Journal of Moldova, 20 (2), 2012, 203-226.

181. (with A. Hashemi) On the Use of Buchberger Criteria in G2V Algorithm for Calculating Gröbner Bases, Programming and Computer Software, vol. 39, no. 2, 2013, 81-90.

182. (with S. Gogilidze, A. Khvedelidze, D. Mladenov, V. Sanadze) Entanglement of spins under strong laser infuence, Physica Scripta, 153, 2013, 014026.

183. (with A. Prokopenya) Simulation of Quantum Error Correction by Means of QuantumCircuit Package, Programming and Computer Software, vol. 39, no. 3, 2013, 143-149.

184. (with A. Hashemi and B. M. Alizadeh) An Involutive Bases Algorithm Incorporating F5 Criterion, Journal of Symbolic Computation, 59, 2013, $1-20$.

185. (with P. Amodio, Yu. A. Blinkov and R. La Scala) On Consistency of Finite Difference Approximations to the Navier-Stokes Equations, in Computer Algebra in Scientific Computing, CASC 2013, V.P. Gerdt, W. Koepff, E. W. Mayr, E. V. Vorozhtsov (Eds.), LNCS, 8136, Springer, Cham, 2013, 46-60.

186. (with A. Prokopenya) Simulation of Quantum Error Correction with Mathematica, in Computer Algebra in Scientific Computing, CASC 2013, V.P. Gerdt, W. Koepff, E. W. Mayr, E. V. Vorozhtsov (Eds.), LNCS, 8136, Springer, Cham, 2013, 116-129.

187. (with A. Khvedelidze and Yu. Palii) Describing the orbit space of global unitary actions for mixed qudit states, Journal of Mathematical Sciences, vol. 200 , no. $6,2014,682-689$.

188. (with D. Michels, D. Lyakhov, G. Sobottka and A. Weber) Lie Symmetry Analysis for Cosserat Rods, in Computer Algebra in Scientifc Computing, $C A S C$ 2014, V.P. Gerdt, W. Koepff, W. M. Seiler, E. V. Vorozhtsov (Eds.), LNCS, 8860, Springer, Cham, 2014, 324-334.

189. (with R. La Scala) Noetherian Quotient of the Algebra of Partial Difference Polynomials and Gröbner Bases of Symmetric Ideals, Journal of Algebra, vol. 423, 2015, 1233-1261.

190. (with A. Khvedelidze and Yu. Palii) Constructing $S U(2) \times U(1)$ orbit space for qutrit mixed states, Journal of Mathematical Sciences, vol. 209, no. $6,2015,878-889$.

191. (with A. A. Gusev, S. I. Vinitsky, V. L. Derbov, A. Góźdź and A. Pędrak) Symbolic algorithm for generating irreducible bases of point groups in the space of $\mathrm{SO}(3)$ group, in Computer Algebra in Scientific Computing, $C A S C$ 2015, V.P. Gerdt, W. Koepff, W. M. Seiler, E. V. Vorozhtsov (Eds.), LNCS, 9301, Springer, Cham, 2015, 166-181.

192. (with D. Michels, D. Lyakhov, G. Sobottka and A. Weber) On Partial Analytical Solution to the Kirchhoff Equation, in Computer Algebra in Scientific Computing, CASC 2015, V. P. Gerdt, W. Koepff, W. M. Seiler, E. V. Vorozhtsov (Eds.), LNCS, 9301, Springer, Cham, 2015, 320-331.

193. (with D. Robertz) Lagrangian constraints and Differential Thomas decomposition, Advances in Applied Mathematics, vol. 72, 2016, 113-138. 
194. (with A. A. Gusev, L. L. Hai, V. L. Derbov, S. I. Vinitsky, O. Chuluunbaatar) Symbolic-Numeric Algorithms for Solving BVPs for a System of ODEs of the Second Order: Multichannel Scattering and Eigenvalue Problems, in Computer Algebra in Scientific Computing / CASC 2016, V. P. Gerdt, W. Koepff, W. M. Seiler, E. V. Vorozhtsov (Eds.), LNCS, 9890, Springer, Cham, 2016, 212-227.

195. (with A. A. Gusev, S. I. Vinitsky, V. L. Derbov, A. Góźdź and A. Pędrak, A. Szulerecka and A. Dobrowolski) Symbolic algorithm for generating irreducible rotational-vibrational bases of point groups, in Computer Algebra in Scientific Computing, CASC 2016, V. P. Gerdt, W. Koepff, W. M. Seiler, E. V. Vorozhtsov (Eds.), LNCS, 9890, Springer, Cham, 2016, 228-242.

196. (with D. Michels, D. Lyakhov, Z. Hossain, I. Riedel-Kruse and A. Weber) On the General, Analytical Solution of the Kinematic Cosserat Equations, in Computer Algebra in Scientific Computing / CASC 2016, V.P. Gerdt, W. Koepff, W. M. Seiler, E. V. Vorozhtsov (Eds.), LNCS, 9890, Springer, Cham, 2016, 367-380.

197. (with A. Khvedelidze and Yu. Palii) On the ring of local unitary invariants for mixed X-states of two qubits, Zapiski Nauchnyh Seminarov POMI, 448, 2016, 107-123.

198. (with A. A. Gusev, S.I. Vinitsky, O. Chuluunbaatar, V.L. Derbov) Symbolic numerical algorithms and programs for the solution of boundaryvalue problems of dynamics of few-body quantum systems, in Proceedings of the 9th International Scientific Conference "Distributed Computer and Communication Networks: Control, Computation, Communications", $D C C N-2016$, V. M. Vishnevskiy, K. E. Samouylov (Eds.), vol. 2, Mathematical Modelling Simulation and Control Problems, Moscow, 2016, $100-108$.

199. (with Yu. A. Blinkov, K. B. Marinov) Computer Algebra Based Discretization of Quaslinear Evolution Equations, Programming and Computer Software, 43 (2), 2017, 84-89.

200. (with P. Amodio, Yu. A. Blinkov and R. La Scala) Algebraic construction and numerical behavior of a new s-consistent difference scheme for the 2D Navier-Stokes equations, Applied Mathematics and Computation, 314, 2017, 408-421.

201. (with R. Bradford, J. H. Davenport, M. England, H. Errami, D. Grigoriev, Ch. Hoyt, M. Kosta, O. Radulescu, T. Sturm and A. Weber) A Case Study on the Parametric Occurrence of Multiple Steady States, in Proceedings of ISSAC 2017, ACM Press, 2017, 45-52.

202. (with D.A. Lyakhov and D. Michels) Algorithmic Verification of Linearizability for Ordinary Differential Equations, in Proceedings of ISSAC 2017, ACM Press, 2017, 285-292.

203. (with A. A. Gusev, O. Chuluunbaatar, G. Chuluunbaatar, S. I. Vinitsky, V.L. Derbov, A. Góźdź) Symbolic Numerical Algorithm for Generating Interpolation Multivariate Hermite Polynomials of High-Accuracy Finite Element Method, in Computer Algebra in Scientific Computing, CASC 2017, V.P. Gerdt, W. Koepff, W. M. Seiler, E. V. Vorozhtsov (Eds.), LNCS, 10490, Springer, Cham, 2017, 135-150.

204. (with A. A. Gusev, O. Chuluunbaatar, G. Chuluunbaatar, S. I. Vinitsky, V. L. Derbov, A. Góźdź) Symbolic Numerical Algorithms for Solving 
the Parametric Self-Adjoint 2D Elliptic Boundary-Value Problem Using High-Accuracy Finite Element Method, in Computer Algebra in Scientific Computing, CASC 2017, V. P. Gerdt, W. Koepff, W. M. Seiler, E. V. Vorozhtsov (Eds.), LNCS, 10490, Springer, Cham, 2017, 151-166.

205. (with D. A. Lyakhov, A. G. Weber and D. L. Michels) Symbolic-Numeric Integration of Dynamical Cosserat Equations, in Computer Algebra in Scientific Computing, CASC 2017, V. P. Gerdt, W. Koepff, W. M. Seiler, E. V. Vorozhtsov (Eds.), LNCS, 10490, Springer, Cham, 2017, 301-312.

206. (with Yu. A. Blinkov and K. B. Marinov) Generation and analysis of a new implicit difference scheme for the Korteveg-de Vries equation, in Proceedings of Mathematical Modeling and Computational Physics, July 3-7, 2017, Dubna, Russia. EPJ Web of Conferences, 173, 2018.

207. (with S. Gusev, S. Vinitsky, O. Chuluunbaatar, G. Chuluunbaatar, V. Derbov, A. Góźdź, P. Krassovitskiy) Interpolation Hermite Polynomials For Finite Element Method, in Proceedings of Mathematical Modeling and Computational Physics, July 3-7, 2017, Dubna, Russia, EPJ Web of Conferences, 173, 2018.

208. (with A. Gusev, S. Vinitsky, O. Chuluunbaatar, G. Chuluunbaatar, V. Derbov, A. Góźdź, P. Krassovitskiy) High-Accuracy Finite Element Method: Benchmark Calculations, in Proceedings of Mathematical Modeling and Computational Physics, July 3-7, 2017, Dubna, Russia EPJ Web of Conferences, 173, 2018.

209. (with Yu. Blinkov, D. Lyakhov and D. Michels) A Strongly Consistent Finite Difference Scheme for Steady Stokes Flow and its Modified Equations, in Computer Algebra in Scientific Computing / CASC 2018, V.P. Gerdt, W. Koepff, W. M. Seiler, E. V. Vorozhtsov (Eds.), LNCS, 11077, Springer, Cham, 2018, 67-81.

210. (with A. Deveikis, A. A. Gusev, S. I. Vinitsky, A. Góźdź and A. Pędrak) Symbolic Algorithm for Generating of Orthonormal Bargmann and Moshinsky Basis for SU(3) group, in Computer Algebra in Scientific Computing, CASC 2018, V.P. Gerdt, W. Koepff, W. M. Seiler, E. V. Vorozhtsov (Eds.), LNCS, 11077, Springer, Cham, 2018, 131-145.

211. (with A. A. Gusev, O. Chuluunbaatar, G. Chuluunbaatar, S. I. Vinitsky, V.L. Derbov, A. Góźdź and P. M. Krassovitskiy) Symbolic-Numerical Algorithms for Solving Elliptic Boundary-Value Problems Using Multivariate Simplex Lagrange Elements, in Computer Algebra in Scientific Computing, CASC 2018, V.P. Gerdt, W. Koepff, W. M. Seiler, E. V. Vorozhtsov (Eds.), LNCS, 11077, Springer, Cham, 2018, 197-213.

212. (with M. Lange-Hegermann, D. Robertz) The MAPLE package TDDS for Thomas decomposition of systems of nonlinear PDEs, Computer Physics Communications, 234, 2019, 202-215.

213. (with D. L. Michels, Yu. A. Blinkov, D. A. Lyakhov) On the consistency analysis of finite difference approximations, Journal of Mathematical Sciences, 240, 5, 2019, 665-677 (Zapiski Nauchnyh Seminarov POMI, 468, 2018, 249-266).

214. (with Xiaojing Zhang, Yury A. Blinkov) Algebraic Construction of a Strongly Consistent, Permutationally Symmetric and Conservative Difference Scheme for 3D Steady Stokes Flow, Symmetry, 11, 269, 2019. 
215. (with Algirdas Deveikis, Alexander Gusev, Vladimir Gerdt, Sergue Vinitsky, Andrzej Góźdź, Aleksandra Pędrak and Cestmir Burdik) Symbolic-Numerical Algorithm for Large Scale Calculations the Orthonormal SU(3) BM Basis, in Computer Algebra in Scientific Computing, $C A S C$ 2019, M. England et al (Eds.), LNCS, 11661, Springer, Cham, 2019, 91-106.

216. (with Yu. A. Blinkov, E. A. Kotkova and I. A. Pankratov) Construction and analysis of a new implicit difference scheme for the 2D Boussinesq paradigm equation, in Computer Algebra in Scientific Computing, CASC 2019, M. England et al (Eds.), LNCS, 11661, Springer, Cham, 2019, $152-163$.

217. (with D. Robertz) Algorithmic approach to strong consistency analysis of finite difference approximations to PDE systems, in Proceedings of ISSAC2019, Beihang University, Beijing, China, July 15-18, 2019, Publications Dept., ACM, New York, USA, 2019, 163-170.

218. (with M. D. Malykh, L. A. Sevastyanov, Yu Ying) On the properties of numerical solutions of dynamical systems obtained using the midpoint method, Discrete \& Continuous Models: Applied Computational Science, vol. 27, no. 1, 2019, 1-22.

219. (with E. A. Kotkova and V. V. Vorob'ev) Teleportation of Bell states performed on quantum computer of IBM, Particles and Nuclei Letters, vol. 16 , no. $6,2019,975-984$.

220. (with D. A. Lyakhov, D. Michels) On the Algorithmic Linearizability for Nonlinear Ordinary Differential Equations, Journal of Symbolic Computation, 98, 2020, 3-22.

221. (with R. Bradford, J. H. Davenport, M. England, H. Errami, D. Grigoriev, Ch. Hoyt, M. Kosta, O. Radulescu, T. Sturm and A. Weber) Identifying the Parametric Occurrence of Multiple Steady States for Biological Networks, Journal of Symbolic Computation, 98, 2020, 84-119.

222. (with K. K. Sharma) Entanglement sudden death and birth effects in two qubits maximally entangled mixed states under quantum channels, International Journal of Theoretical Physics, 59, 2020, 403-414.

\subsection{Communications of Joint Institute for Nuclear Research}

Since 1999 available at www1.jinr.ru/Preprints/Preprints_rus.html.

1. (with V.A. Meshcheryakov) New Type of Sum Rules for $p$ N-Scattering in Subthreshold Region, JINR R2-7222, Dubna, 1973.

2. (with V. A. Meshcheryakov) Local Study of Rest Points of the Chew-Low Type Equation, JINR R2-7976, Dubna, 1974.

3. (with V.E. Aleinikov, M. A. Ignatenko and V. I. Zovbun) Radiation Situation in the Area of the Canal of Slow Beam Which Is Extracted from the $10 \mathrm{GeV}$ Synchrophasotron, JINR 16-8583, Dubna, 1975.

4. Local Uniformization of Amplitude of Elastic Hadron-Hadron Scattering, JINR 2-9709, Dubna, 1976.

5. (with O. V. Tarasov and D. V. Shirkov) Analytical Calculations by Computer in Physics and Mathematics, JINR R2-11547, Dubna, 1978.

6. (with F. Kh. Abdullaev and J.S. Vaklev) The Role of Nonlinearity in a Model with a Strange Attractor, JINR R4-80-446, Dubna, 1980. 
7. (with A. Yu. Zharkov) Elementary Fraction Decomposition of Rational Functions in System REDUCE-2, JINR R5-82-187, Dubna, 1982.

8. (with N. A. Kostov and A. B. Shvachka) Investigation of Nonlinear Water Waves Using Computer Algebra System REDUCE-2, JINR E11-83-750, Dubna, 1983.

9. (with A.B. Shvachka and A. Yu. Zharkov) Classification of Integrable High-Order KdV-Like Equations, JINR R5-84-489, Dubna, 1984.

10. (with A. Yu. Zharkov) On Asymptotic Expansion of General Solution of Chew-Low Equations, JINR R5-84-431, Dubna, 1984.

11. (with N. A. Kostov, P. P. Raychev and R. P. Russev) Calculation of the Matrix Elements of the Hamiltonian of the Interacting Vector Boson Model Using Computer Algebra. Basic Concepts of the Interacting Vector Boson Model and Matrix Elements of the SU(3)-Quadrupole Operator, JINR E4-85-262, Dubna, 1985.

12. (with N. A. Kostov, P. P. Raychev and R. P. Russev) Calculation of the Matrix Elements of the Hamiltonian of the Interacting Vector Boson Model Using Computer Algebra. Matrix Elements of the Hamiltonian and Some U(6)-Clebsh-Gordon Coefficients, JINR E4-85-263, Dubna, 1985.

13. (with N. A. Kostov, P. P. Raychev and R. P. Russev) Calculation of the Matrix Elements of the Hamiltonian of the Interacting Vector Boson Model Using Computer Algebra. Matrix Elements of the HamiltonianAnalytical Results, JINR E4-85-264, Dubna, 1985.

14. (with A.S. Ilchev, V.K. Mitrjushkin and A. M. Zadorozhny) On the Phase Structure of Lattice SU(2) Gauge-Higgs Theory, JINR E2-85-104, Dubna, 1985.

15. (with M. G. Meshcherykov and D. V. Shirkov) Computers in Theoretical Physics, JINR R2-86-848, Dubna, 1986.

16. (with A. Yu. Zharkov) Solving the Polynomial Equations Arising in Classification of Integrable Coupled KdV-like Systems, JINR R5-89-231, Dubna, 1989.

17. (with N. A. Kostov, Z. T. Kostova and I. P. Yudin) Algebraic-Numeric Calculations of Proton Trajectories in Bending Magnets of Synchrotron Accelerator, JINR E11-89-755.

18. (with L. M. Berkovich, Z. T. Kostova and M. L. Nechaevsky) Computer Algebra Generating Related 2nd Order Linear Differential Equation, JINR E5-90-509, Dubna, 1990.

19. (with A. Yu. Zharkov) Algorithms for Investigating Integrability of Quasilinear Evolution Systems with Non-degenerated Main Matrix, JINR R5-91-225, Dubna, 1991.

20. (with P. Tiller) A Reduce Program for Symbolic Computation of Puiseux Expansions, JINR E5-91-401, Dubna, 1991.

21. (with W. Lassner) Verifying Isomorphisms of Finite Dimensional Lie Algebras by Gröbner Basis Technique, JINR E5-92-145, Dubna, 1992.

22. Computer Algebra Methods in Investigation of Integrability of Nonlinear Evolution Equations, JINR 11-92-258, Dubna, 1992. 


\subsection{Preprints and technical reports}

1. Computer Algebra and Nonlinear Equations: Recent Achievements, Publication IT-270, Laboratoire d'Informatique Fondamentale de Lille, Lille, 1995.

2. (with Yu. A. Blinkov) Involutive Polynomial Bases, Publication IT-271, Laboratoire d'Informatique Fondamentale de Lille, Lille, 1995.

\subsection{Prepared articles}

1. (with D. Robertz, Yu. A. Blinkov) Strong Consistency and Thomas Decomposition of Finite Difference Approximations to Systems of Partial Differential Equations, 2020, arXiv:2009.01731

2. (with M. D. Malykh, L. A. Sevastianov, Yu Ying) On conservative difference schemes for the many-body problem, 2020, arXiv:2007.01170.

3. (with Yu. A.Blinkov) Compact and Computationally Efficient Involutive Bases.

4. (with K. K. Sharma, P. V. Gerdt) Milestone Developments in Quantum Information and No-Go Theorems.

\section{Additional materials}

\subsection{Last video of Vladimir Gerdt}

1. Compact involutive monomial bases. Joint scientific seminar of the Institute of Applied Mathematics \& Communications Technology, 18 Nov. 2020. URL: https://events.rudn.ru/event/102.

2. Computer algebra based discretizations of incompressible Navier-Stokes equations. Joint scientific seminar of the Institute of Applied Mathematics \& Communications Technology, 25 Nov. 2020. URL: https://events . rudn.ru/event/103.

\subsection{Memorial photo galleries of Vladimir Gerdt}

1. Collection of Yu. A. Blinkov.

URL: https://disk.yandex.ru/d/dTxdWyb2_TBAYg

2. Collection of V.F. Edneral.

URL: https://yadi.sk/d/tdFNE877AzQAcw

\section{Acknowledgments}

The author thanks A. A. Bogolyubskaya, A. V. Korolkova and M. D. Malykh, who participated in editing the manuscript. The subsections $4.2,4.3,4.4$ is written in collaboration with Yu. A. Blinkov (Saratov State University) and M. D. Malykh (RUDN University), the subsection 4.5, 4.6 is written in collaboration with A. Khvedelidze (JINR, Dubna). List of the published works of Vladimir Gerdt was compiled by A. A. Bogolyubskaya. 


\section{References}

[1] C. Riquier, Les Systèmes d'Equations aux Dérivées Partielles. Paris: Gauthier-Villars, 1910.

[2] M. Janet, "Systèmes d'équations aux dérivées partielles," Journals de mathématiques, 8e série, vol. 3, pp. 65-151, 1920.

[3] J. Thomas, Differential systems. New York: American Mathematical Society, 1937.

[4] D. Robertz, Formal algorithmic elimination for PDEs. Springer, 2014.

[5] D. Cox, J. Little, and D. O'Shea, Ideals, varieties, and algorithms, 3rd ed. Springer, 2007.

[6] F. S. Macaulay, "Some properties of enumeration in the theory of modular systems," Proceedings of the London Mathematical Society, vol. s2-26, no. 1, pp. 531-555, 1927. DOI: $10.1112 / \mathrm{plms} / \mathrm{s} 2-26.1 .531$.

[7] A. Y. Zharkov and Y. A. Blinkov, "Involution approach to solving systems of algebraic equations," in Proceedings of the 1993 International IMACS Symposium on Symbolic Computation. Laboratoire d'Informatique Fondamentale de Lille, France, 1993, pp. 11-16.

[8] A. Y. Zharkov, "Involutive polynomial bases: General case," in Preprint JINR E5-94-224. Dubna, 1994.

[9] A. Y. Zharkov and Y. A. Blinkov, "Algorithm for constructing involutive bases of polynomial ideal," in International Conference on Interval and Computer-Algebraic Methods in Science and Engineering. St-Petersburg, 1994, pp. 258-260.

[10] A. Y. Zharkov and Y. A. Blinkov, "Involutive bases of zero-dimensional ideals," in Preprint JINR E5-94-318. Dubna, 1994.

[11] V. P. Gerdt, "Gröbner bases and involutive methods for algebraic and differential equations," in Computer Algebra in Science and Engineering. Singapore: World Scientific, 1995, pp. 117-137.

[12] J. Apel, "A Gröbner approach to involutive bases," Journal of Symbolic Computation, vol. 19, no. 5, pp. 441-458, 1995.

[13] V. P. Gerdt and Y. A. Blinkov, "Involutive polynomial bases," in Publication IT-95-271. Laboratoire d'Informatique Fondamentale de Lille, 1995.

[14] V. P. Gerdt and Y. A. Blinkov, "Involutive Bases of Polynomial Ideals," in Preprint-Nr.1/1996. Naturwissenschaftlich-Theoretisches Zentrum, University of Leipzig, 1996.

[15] V. P. Gerdt, "Gröbner bases and involutive methods for algebraic and differential equations," Mathematical and computer modelling, vol. 25, no. 8-9, pp. 75-90, 1997. DOI: 10.1016/S0895-7177 (97)00060-5.

[16] V. P. Gerdt and Y. A. Blinkov, "Involutive bases of polynomial ideals," in Preprint JINR E5-97-3. Dubna, 1997. 
[17] V. P. Gerdt, "Involutive divisions in mathematica: Implementation and some applications," in Proceedings of the Rhein Workshop on Computer Algebra. Institute for Algorithms and Scientific Computing, GMD-SCAI, 1998, pp. 74-91.

[18] V. P. Gerdt and Y. A. Blinkov, "Involutive divisions of monomials," Programming and Computer Software, vol. 24, no. 6, pp. 283-285, 1998.

[19] Y. A. Blinkov, "Division and algorithms in the ideal membership problem [Deleniye i algoritmy v zadache o prinadlezhnosti k idealu]," Izvestija Saratovskogo universiteta, vol. 1, no. 2, pp. 156-167, 2001, In Russian.

[20] K. Lipnikov, G. Manzini, and M. Shashkov, "Mimetic finite difference method," Journal of Computational Physics, vol. 257, pp. 1163-1227, 2014. DOI: $10.1016 / j \cdot j c p .2013 .07 .031$.

[21] B. Koren, R. Abgrall, P. Bochev, J. Frank, and B. Perot, "Physicscompatible numerical methods," Journal of Computational Physics, vol. 257, no. Part B, pp. 1039-1524, 2014. DOI: 10 . $1016 /$ j · jcp . 2013.10.015.

[22] M. Shashkov, Conservative finite difference methods. Boca Raton: CRC Press, 1996.

[23] D. Arnold, P. Bonchev, R. Lehoucq, R. Nikolaides, and M. Shashkov, Compatible spatial discretizations. Springer-Verlag, Berlin, 2006.

[24] L. B. da Veiga, K. Lipnikov, and G. Manzini, The mimetic finite difference method for elliptic problems. Springer, 2014, vol. 11.

[25] J. E. Castillo and G. F. Miranda, Mimetic discretization methods. Chapman and Hall/CRC, 2013.

[26] V. P. Gerdt, D. Robertz, and Y. A. Blinkov, "Strong Consistency and Thomas Decomposition of Finite Difference Approximations to Systems of Partial Differential Equations," 2020. arXiv: 2009.01731 [cs.SC].

\section{For citation:}

V.F.Edneral, In Memory of Vladimir Gerdt, Discrete and Continuous Models and Applied Computational Science 29 (4) (2021) 306-336. DOI: 10.22363/2658-4670-2021-29-4-306-336.

\section{Information about the authors:}

Edneral, Viktor F. - Candidate of Physical and Mathematical Sciences, Senior Researcher of Skobeltsyn Institute of Nuclear Physics of Lomonosov Moscow State University; Assistant professor of Department of Applied Probability and Informatics of Peoples' Friendship University of Russia (RUDN University) (e-mail: edneral@theory.sinp.msu.ru, edneral-vf@rudn.ru, phone: +7(495)9522823, ORCID: https://orcid.org/0000-0002-5125-0603, ResearcherID: E-2138-2012, Scopus Author ID: 6602198073) 
УДК 92:51

PACS 01.65.+g

DOI: $10.22363 / 2658-4670-2021-29-4-306-336$

\section{Памяти Владимира Гердта}

\section{В. Ф. Еднерал}

${ }^{1}$ НИИ ядерной физики имени Д. В. Скобельцына МГУ Ленинские горы, д. 1 (2), Москва, 119991, Россия

${ }^{2}$ Российский университет дружбы народов ул. Миклухо-Маклая, д. 6, Москва, 117198, Россия

Настоящая статья - мемориальная, она посвящена памяти руководителя научного центра вычислительных методов в прикладной математике РУДН, профессора В. П. Гердта, чей уход стал невосполнимой потерей для научного центра и всего сообщества компьютерной алгебры. В статье приведены биографические сведения о В. П. Гердте, рассказано о его вкладе в развитие компьютерной алгебры в России и мире. В конце приведены личные воспоминания автора о В. П. Гердте.

Ключевые слова: компьютерная алгебра, квантовые вычисления, миметические методы, методы полиномиальной компьютерной алгебры 